Check for updates

Cite this: RSC Adv., 2017, 7, 34077

Received 13th March 2017 Accepted 30th June 2017

DOI: $10.1039 / c 7 r a 02985 c$

rsc.li/rsc-advances

\section{A graphene oxide surface-molecularly imprinted polymer as a dispersive solid-phase extraction adsorbent for the determination of cefadroxil in water samples}

\begin{abstract}
Xinwei Chen and Nengsheng Ye (DD *
In this work, a graphene oxide (GO)-functionalized molecularly imprinted polymer (MIP) (GO-MIPs) was prepared using a non-covalent molecular imprinting approach and applied to dispersive solid-phase extraction (DSPE) coupled with ultra-high-performance liquid chromatography with photodiode array detection (UPLC-PDA) to sensitively detect cefadroxil (FAD) in aqueous solution. FAD was used as a template to synthesize GO-MIPs, with 2-(trifluoromethyl) acrylic acid (TFMAA) and ethylene glycol dimethacrylate (EGDMA) as the monomer and cross-linker, respectively, in methanol. The GO-MIPs was eluted with acetic acid-methanol (20/80, v/v) and then characterized by scanning electron microscopy (SEM), transmission electron microscopy (TEM), thermal gravimetric analysis (TGA) and Raman spectroscopy. The selectivity of the GO-MIPs versus a graphene oxide-functionalized non-imprinted polymer (GO-NIPs) was confirmed based on the selectivity factors (SFs) using cefixime, cefoxitin sodium and ceftiofur hydrochloride as structural analogues of FAD. The efficiency of the synthesized GO-MIPS was evaluated through application of the proposed DSPE procedure. The influencing factors of DSPE, such as the sample $\mathrm{pH}$, extraction time, desorption reagents and desorption time, were optimized, and the analytical performance of the developed DSPE-UPLC-PDA method was evaluated under the optimized conditions. Good linearity was obtained over $0.04-6.0 \mu \mathrm{g} \mathrm{mL}^{-1}\left(R^{2}=0.9979\right)$, with a detection limit $(S / N=3)$ of $0.01 \mu \mathrm{g} \mathrm{mL}^{-1}$. Finally, the developed method was applied to determine the concentration of FAD in water samples, and the spiked average recoveries ranged between $72.5 \%$ and $104.8 \%$. The GO-MIPs served as good carriers for the selective adsorption of FAD and showed promise for the preconcentration of FAD in complex samples.
\end{abstract}

\section{Introduction}

Consumers have recently shown increasing awareness of food safety. Public control agencies and the food industry work to ensure high levels of control of food for human consumption. Among man-made contaminants, antibiotics are of particular concern because of the development of antibiotic resistance, which has long been attributed to their overuse in human medicine and recently to their use in animal breeding with possible contamination of foodstuffs. ${ }^{1}$ Therefore, antibiotic residues are frequently monitored in a wide range of sample matrices, such as foodstuffs and their surroundings.

The term antibiotic refers to a diverse range of chemical substances that possess antibacterial activity and can be either broad or narrow spectrum. ${ }^{2} \beta$-Lactam antibiotics constitute one of the most widely used antimicrobial drugs in veterinary medicine, especially in the treatment and prevention of

Department of Chemistry, Capital Normal University, Beijing 100048, P. R. China. E-mail:yensh@cnu.edu.cn bacterial infections (respiratory, urinary, mammary gland and skin infections) in dairy cattle. This group of antibiotics can be classified into several groups according to their structural characteristics: penicillins, cephalosporins, and, more recently, carbapenems. ${ }^{3}$ Among these antibiotics, cephalosporins are generally unstable in solution and in the solid state. ${ }^{4}$ Cephalosporins are frequently used to treat infections caused by Grampositive and Gram-negative bacteria. Because cephalosporins with a $\beta$-lactam ring interfere with the synthesis of the bacterial cell wall, they are one of the most effective antimicrobial agents. ${ }^{5,6}$ In fact, these cephalosporins have been used in livestock farming as prophylactic and therapeutic agents and as feed additives for growth promotion. ${ }^{7}$ At the same time, cephalosporin residues pose a risk to human health because they can cause allergic reactions in hypersensitive individuals and can lead to the emergence of drug-resistant bacteria. Moreover, cephalosporins are the most prescribed for example cefadroxil, cefixime, cefoxitin and so on. ${ }^{\mathbf{8} 9}$ To probe the potential benefits and adverse effects of cephalosporin consumption more efficiently, a suitable sample pretreatment method is necessary to 
decrease matrix interferences and facilitate the detection of cephalosporins at trace levels. Dispersive solid-phase extraction (DSPE) is based on SPE methodology, but the sorbent is added directly to the sample solution, followed by centrifugation after extraction. ${ }^{10,11}$ Compared with conventional SPE methods, ${ }^{12}$ the main advantage of DSPE is the effective increase in active surface area between the analytes and sorbent. Thus, a novel molecularly imprinted polymer (MIPs) with a graphene oxide (GO)-functionalized surface was synthesized as an adsorbent for coupling with DSPE to preconcentrate cefadroxil (FAD). The advantage of GO-MIPs particles is to demonstrate the good ability for specific selective extraction of FAD in environmental water samples.

Graphene reportedly possesses a high theoretical specific surface area $\left(2630 \mathrm{~m}^{2} \mathrm{~g}^{-1}\right)$, which suggests a high sorption capacity. In addition to graphene, GO, a two-dimensional monolayer of carbon atoms arranged in a honeycomb lattice has attracted much attention in recent years because of its ultrahigh specific surface area. ${ }^{\mathbf{1 3 , 1 4}} \mathrm{GO}$, a precursor to graphene after reduction, contains a range of reactive hydroxyl, carboxyl, and epoxide functional groups on its basal plane, making it a good candidate for use in chemical functionalization reactions, ${ }^{15,16}$ including the synthesis of MIPs on the surface of GO. ${ }^{17,18}$ Moreover, GO sheets can greatly improve the chemical and physical properties when incorporated in composite materials. ${ }^{\mathbf{1 9 , 2 0}}$ Due to its ultrahigh surface area and excellent chemical properties, GO has been explored as a supporting material of adsorbents (MIPs) for the preconcentration of small molecules. ${ }^{17,21}$

Molecular imprinting is a new molecular recognition technology that, compared with natural receptors, not only offers specific molecular recognition sites for molecular targets but also has distinct advantages such as high chemical stability, easy and inexpensive preparation, and potential reusability. ${ }^{22,23}$ Molecular imprinting has been widely recognized as a potential technique for the synthesis of tailor-made recognition materials through the formation of a polymer network around a template molecule. Owing to these fantastic properties, MIPs have been widely used in the fields of biosensors, chromatographic separation, catalysis, drug delivery and SPE. ${ }^{24-26}$ MIPs, which are synthetic polymers that enable simultaneous pretreatment and enrichment, exhibit high selectivity for and specific recognition of a given analyte or a group of structurally related species. MIPs are often coupled with SPE as molecularly imprinted SPE (MISPE), which has high selectivity and specificity and a strong separation capacity. ${ }^{27}$ Because of its higher chemical stability and selectivity and more facile preparation, MISPE has been widely applied in food analysis, ${ }^{28,29}$ biochemical analysis, ${ }^{30,31}$ environmental analysis, ${ }^{32}$ etc. Although MIPs have unique advantages, challenges involving the imperfect removal of template molecules and recognition site destruction remain after polymerization. ${ }^{33}$ Therefore, surface imprinting has emerged to overcome such drawbacks, in which recognition sites are formed on the material surface. ${ }^{34}$ Some studies have reported molecular imprinting methods that utilize the surface of support substrates, including GO and nanoalumina etc. ${ }^{35}$ The imprinted film prepared on the surface of GO via imprinting can enable the rebinding and extraction of the template molecules, increase the response kinetics, and improve access to the surface binding sites. ${ }^{36}$

The present study built upon our previous research to prepare a surface-imprinted polymer through the selfpolymerization of FAD on the surface of GO. ${ }^{18}$ GO-MIPs-DSPE combined with ultra-high-performance liquid chromatography (UPLC) was used for the preconcentration and determination of FAD. The morphology of the surface-imprinted polymer was characterized using several techniques. The chromatographic conditions were successfully optimized using a gradient elution method. The DSPE optimization and analyte stability are also discussed, and the accuracy and precision of the UPLC analysis and method detection limits are described. The GO-MIPsDSPE-UPLC method was validated using river and tap water samples.

\section{Experimental}

\subsection{Reagents and materials}

GO was purchased from XFNano Materials Tech. Co., Ltd. (Nanjing, China). FAD, cefixime (CFM), cefoxitin sodium (FOX), ceftiofur hydrochloride (EFT) (chemical structures shown in Fig. 1) ethylene glycol dimethacrylate (EGDMA) and 2-(trifluoromethyl) acrylic acid (TFMAA) were obtained from SigmaAldrich (Shanghai, China). N,N-Dimethyl formamide (DMF) and acetic acid were purchased from the Beijing Chemical Plant (Beijing, China). 2,2-Azobisisobutyronitrile (AIBN) was supplied by the Aladdin Reagent Co., Ltd. (Shanghai, China). Deionized water (18.2 M $\Omega$ ) was obtained from a Milli-Q system (Billerica, MA. USA). All other reagents were of analytical grade and used without further purification. All solutions were filtered through filters with a $0.22 \mu \mathrm{m}$ pore size (Tianjin, China). Chromatographic-grade methanol, acetonitrile and acetone were purchased from Merck Co. (Darmstadt, Germany).

To prepare the surfactant-stabilized GO dispersions, $120.0 \mathrm{mg}$ of GO was sonicated in $40 \mathrm{~mL}$ of DMF for $2 \mathrm{~h}$, after which the GO was evenly dispersed in the DMF at a concentration of $3.0 \mathrm{mg} \mathrm{mL}^{-1} \mathrm{GO}$.

\subsection{Instruments and chromatographic conditions}

All the chromatographic analyses were performed on a Waters Acquity UPLC ${ }^{\mathrm{TM}}$ system with a quaternary solvent manager,
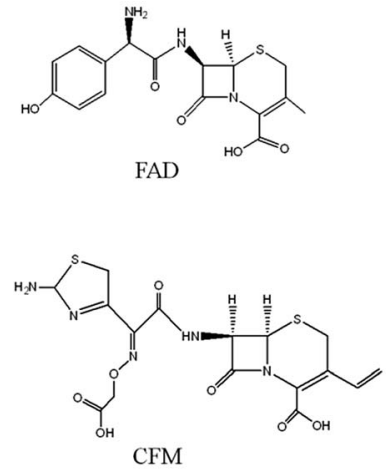

Fig. 1 Chemical structures of the four cephalosporins investigated in this work. 
a sample manager and a photodiode array (PDA) detector. A Waters UPLC BEH C18 column $(50 \mathrm{~mm} \times 2.1 \mathrm{~mm}$ i.d., $1.7 \mu \mathrm{m})$ was used as the analytical column. System control and chromatographic data acquisition were carried out using Empower software (Waters Technologies, USA). Gradient elution was performed with acetonitrile (solvent A) and a sodium acetateacetic acid (NaAC-HAC) buffer solution ( $\mathrm{pH}=3.4$, solvent $\mathrm{B}$ ). The elution program was as follows: $0.0-2.5 \mathrm{~min}$, solvent $\mathrm{A} /$ solvent B (5/95, v/v); 2.5-3.0 min, solvent A/solvent B (25/75, v/ $\mathrm{v})$; and 3.0-5.0 $\mathrm{min}$, solvent A/solvent B (5/95, v/v). The flow

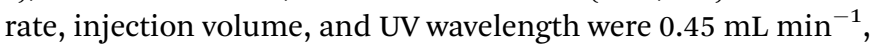
$10 \mu \mathrm{L}$, and $270 \mathrm{~nm}$, respectively. All of the mobile phases were filtered through a $0.22 \mu \mathrm{m}$ filter membrane and degassed for $30 \mathrm{~min}$ before use. The centrifuge was purchased from Sigma (3K15, Germany), and centrifugation was performed at $25{ }^{\circ} \mathrm{C}$.

Scanning electron microscopy (SEM) images were obtained using an S-4800 field scanning electron microscope (Hitachi, Japan) operating at $15 \mathrm{kV}$. Transmission electron microscopy (TEM) images were obtained using a JEM 1200 EX transmission electron microscope (JEOL, Japan). Nitrogen adsorption/ desorption isotherms images were measured by MicroActive for ASAP 2460 (Micromeritics, USA). Raman spectra were collected using a Raman spectrometer (Renishaw, UK) with a $633 \mathrm{~nm}$ excitation wavelength. Thermal gravimetric analysis (TGA) was conducted on an HCT-1 instrument (Beijing Henven Scientific Instrument Factory, Beijing) from room temperature to $800{ }^{\circ} \mathrm{C}$ at a heating rate of $10{ }^{\circ} \mathrm{C} \mathrm{min}^{-1}$ under nitrogen flow.

\subsection{Preparation of stock solutions and real samples}

Stock solutions of FOX, CFM, FAD and EFT were prepared in acetonitrile and stored at $4{ }^{\circ} \mathrm{C}$ until use. Working solutions of

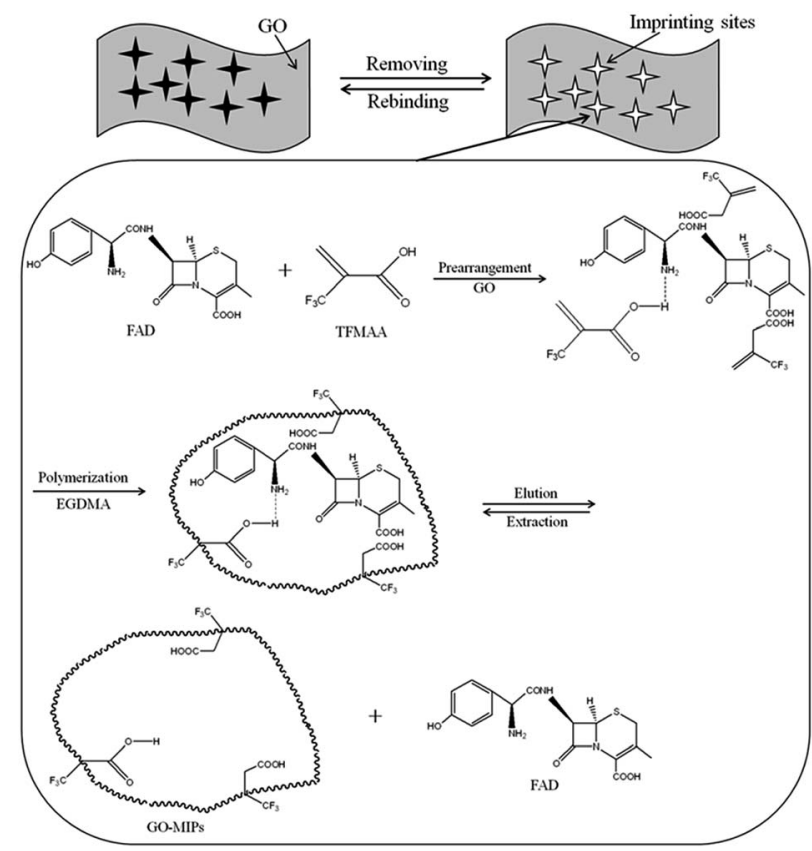

Fig. 2 Illustration of the preparation of graphene oxide functionalized surface-imprinted polymer (GO-MIPs). different concentrations were freshly prepared by appropriate dilution of the stock solution with deionized water. River water samples were collected from the Huaisha River (Beijing, China), and tap water samples were collected from a water tap in our laboratory (Beijing, China). The water samples were filtered through a $0.22 \mu \mathrm{m}$ filter before use without any additional pretreatment. After filtration through a $0.22 \mu \mathrm{m}$ membrane filter, the sample solution was subjected to GO-MIPs-DSPEUPLC.

\subsection{Synthesis of GO-MIPs}

GO-MIPs were prepared by the non-covalent approach according to the developed strategy, with slight modifications. A MIPs was prepared using FAD as the template molecule and TFMAA as the functional monomer by adding $1 \mathrm{mmol}$ of FAD and $4 \mathrm{mmol}$ of TFMAA to $5 \mathrm{~mL}$ of methanol. After the FAD had completely dissolved, $20 \mathrm{~mL}$ of a GO dispersion $\left(3 \mathrm{mg} \mathrm{mL}^{-1}\right)$, $20 \mathrm{mmol}$ of the cross-linking agent EGDMA and $40 \mathrm{mg}$ of the initiator AIBN were added. The mixture was degassed by sonication for $10 \mathrm{~min}$ and then bubbled with nitrogen for $10 \mathrm{~min}$. Degassing and bubbling were repeated three times. Next, the temperature was held at $60{ }^{\circ} \mathrm{C}$ for $24 \mathrm{~h}$ for polymerization. Polymerization occurred via non-covalent binding, including ionic interactions, hydrogen bonds, and $\pi-\pi$ stacking, making preparation of the FAD GO-MIPs possible (schematic of the GO-functionalized MIPs procedure is shown in Fig. 2). The effective charge transfer between the template monomer and GO agreed with the Raman spectral analysis. After the reaction, the bulk GO-MIPs were ground to obtain a suitable size range of the GO-MIPs particles with different size ranges $(20-80 \mu \mathrm{m}, 80-$ $100 \mu \mathrm{m}$ and 100-140 $\mu \mathrm{m})$. The resulting particles were washed with acetic acid-methanol (20/80, v/v) until FAD was no longer detected in the eluent by UPLC-PDA. The particles were then washed with distilled water and dried to a constant weight at $60{ }^{\circ} \mathrm{C}$.

Reference GO-functionalized-non-imprinted polymers (GONIPs) were prepared using the same procedure but without the addition FAD as the template during polymerization.

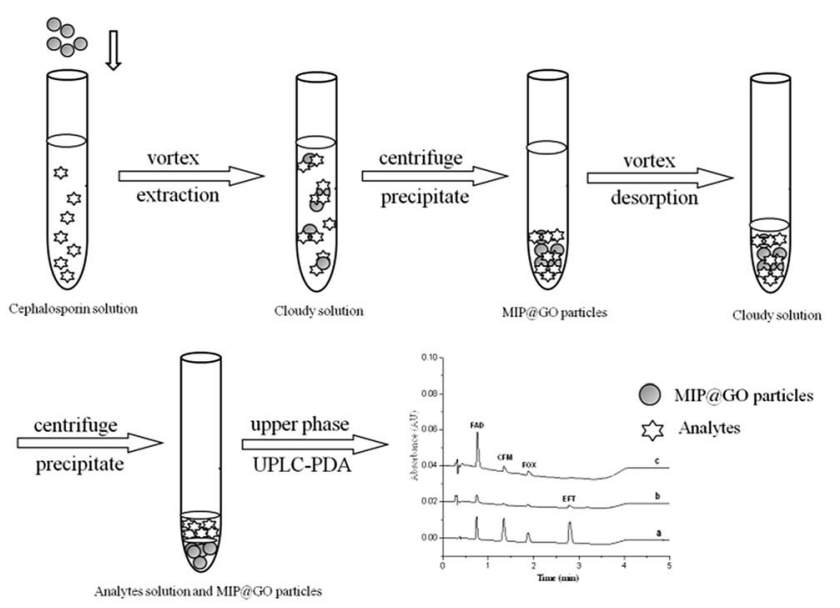

Fig. 3 Schematic of GO-MIPs-DSPE combined with UPLC. 


\subsection{Dispersive solid-phase extraction}

Ten milliliter samples spiked with $1 \mu \mathrm{g} \mathrm{mL}{ }^{-1}$ FAD, CFM, FOX and EFT were adjusted to $\mathrm{pH} 6.0$ and transferred to a centrifuge tube containing $100 \mathrm{mg}$ of GO-MIPs particles. The mixture was extracted for $40 \mathrm{~min}$ under agitation at $1500 \mathrm{rpm}$ and subsequently centrifuged for $5 \mathrm{~min}$ at $6000 \mathrm{rpm}$. The upper phase was discarded. Then, the mixture was desorbed for $30 \mathrm{~min}$ by $1 \mathrm{~mL}$ of acetic acid-methanol $(20 / 80, \mathrm{v} / \mathrm{v})$ under agitation at $1500 \mathrm{rpm}$ and centrifuged for $5 \mathrm{~min}$ at $6000 \mathrm{rpm}$. Finally, acetic acidmethanol layer was filtered through a $0.22 \mu \mathrm{m}$ filter membrane and analyzed by UPLC-PDA. The whole extraction process is shown in Fig. 3.

\section{Results and discussion}

\subsection{Characterization of GO, GO-NIPs and GO-MIPs}

In this research, GO-MIPs and GO-NIPs were synthesized on the surface of GO. GO-MIPs with different size ranges were obtained, and GO-MIPs particles in the size range of $80-100 \mu \mathrm{m}$ were chosen for the further study because of its higher adsorption capability. These materials were characterized with SEM, TEM, TGA and Raman spectroscopy.

3.1.1 SEM and TEM. The morphology and structure of the GO, GO-NIPs, GO-MIPs were analyzed and characterized by SEM. As shown in Fig. 4a, the GO sheets exhibited a layered structure with a fairly irregular surface that was thin, wrinkled, and typically curved, which is typical of GO. After polymerization, the surface of the GO-NIPs (Fig. 4b) and GO-MIPs (Fig. 4c and d) composites became rough and new shapes appeared. Compared with the morphology of the GO-NIP particles, the GO-MIPs particles showed a high degree of cross-linking because the target molecules provided sites for the polymerization reaction. ${ }^{37}$ Fig. $4 \mathrm{~b}-\mathrm{d}$ shows SEM images of the GO-NIPS

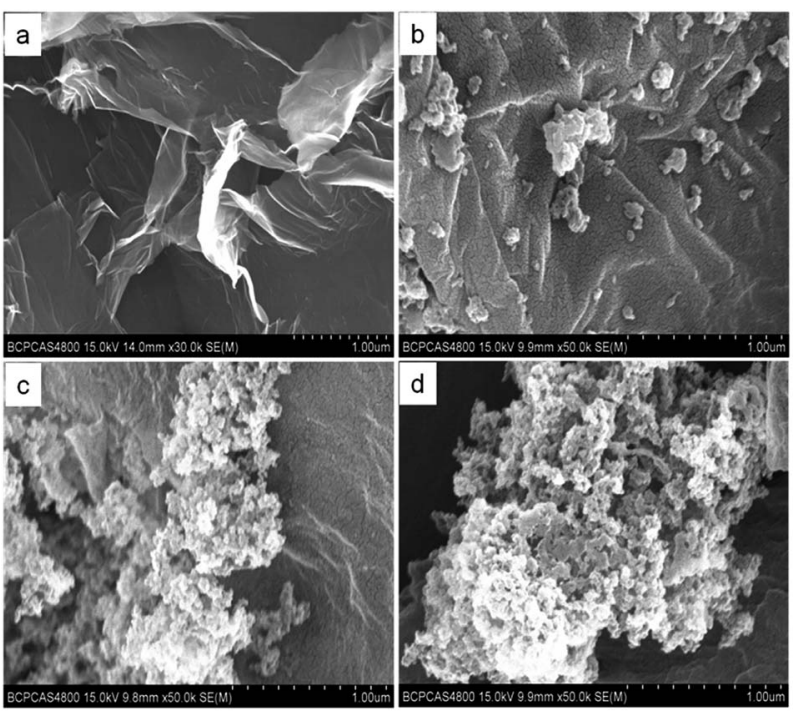

Fig. 4 SEM images of GO (a), GO-NIPs (b), GO-MIPs before template elution (c), and GO-MIPs after template elution (d). Conditions: acceleration voltage, $15 \mathrm{kV}$; magnification, (a) $\times 30000$; (b-d) $\times 50000$. and GO-MIPs before and after template elution. Surface area and pore volume calculation by Brunauer-Emmett-Teller (BET) (Fig. 5d) was further investigated for the GO-MIPs before and after template elution. The surface area and pore volume of GOMIPs before template elution is $7.96 \mathrm{~m}^{2} \mathrm{~g}^{-1}$ and $0.02 \mathrm{~m}^{3} \mathrm{~g}^{-1}$, respectively. The surface area and pore volume of GO-MIPs after template elution is $238.19 \mathrm{~m}^{2} \mathrm{~g}^{-1}$ and $0.71 \mathrm{~m}^{3} \mathrm{~g}^{-1}$, respectively. The results showed that the surface area of GOMIPs is far less than that of graphene and GO, and the GO-MIPs particles synthesized on the surface of GO resulted the reduction of surface area. For the increase of surface area of GO-MIPs after template elution, this may be because the GO-MIPs presented a homogeneous and dense surface with no obvious porous structure before elution but had more pores after elution. This porous structure could promote the mass transfer of FAD between the GO-MIPs particles and sample solution.

The morphological structure of GO and the GO-MIPs was examined by TEM. From the TEM image in Fig. 5a, GO was very thin and contained some wrinkles, similar to wrinkled paper. These wrinkles may be important for preventing the aggregation of GO and maintaining a high surface area. ${ }^{37}$ As observed in Fig. $5 \mathrm{~b}$ and c, the approximate GO sheets were decorated by the MIPs, which indicated that the imprinted sites were generated in the GO-MIPs hybrid and that the GO-MIPs particles had been successfully synthesized.

3.1.2 TGA. The TGA weight loss curves for GO and the GOMIPs particles were investigated. As shown in Fig. 6a, the weight of GO decreased sharply between $100{ }^{\circ} \mathrm{C}$ and $200{ }^{\circ} \mathrm{C}(40 \%$ total weight loss), which was attributed to the removal of labile oxygen-containing functional groups $(-\mathrm{OH}$ and $-\mathrm{COOH})$ from GO. Therefore, GO was not thermally stable. As shown in Fig. 6b, the weight of the GO-MIPs particles decreased rapidly between $350{ }^{\circ} \mathrm{C}$ and $450{ }^{\circ} \mathrm{C}$, which might have been due to the thermal decomposition of the polymer. After the polymerization reaction, the GO-MIPs composites appeared to be more effective than GO at enhancing the thermal stability of the GO
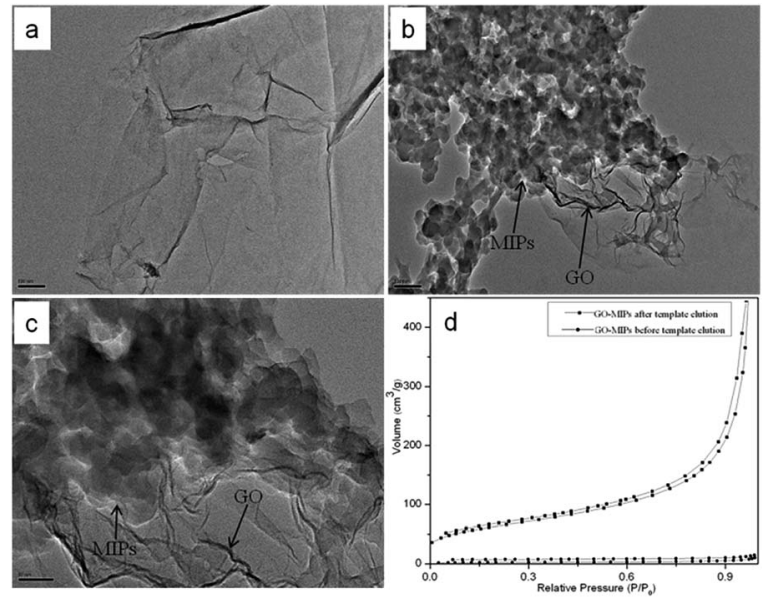

Fig. 5 TEM images of GO (a) and GO-MIPs ( $b$ and $c)$. Conditions: acceleration voltage, $300 \mathrm{kV}$; magnification, (a) $\times 20000$; (b) $\times 20$ 000; (c) $\times 50$ 000. (d) Nitrogen adsorption/desorption isotherms of GO-MIPs before and after template elution. 
sheets. ${ }^{38}$ Due to the different thermal stability of GO and the GO-MIPs composites, the TGA measurements showed that the MIPs layer was successfully adhered to the GO surface.

3.1.3 Raman spectroscopy. Raman spectroscopy is a useful tool to characterize carbon materials with high Raman intensities, especially for determining the electronic states and distinguishing ordered and disordered crystal structures. In general, the Raman spectra of carbonaceous materials exhibit a D-band peak at approximately $1340 \mathrm{~cm}^{-1}$ and a G-band peak at approximately $1600 \mathrm{~cm}^{-1}$, which are due to the doubleresonance excitation of phonons close to the $K$ point in the Brillouin zone. $^{39}$ The D-band and G-band peaks represent disordered $\mathrm{sp}^{3}$ carbons and ordered $\mathrm{sp}^{2}$ crystalline graphite-like structures, respectively. Fig. 7 shows that the GO, GO-NIPs and GO-MIPs samples all produced two high peaks at approximately $1330 \mathrm{~cm}^{-1}$ and $1600 \mathrm{~cm}^{-1}$ due to their carbonaceous structures. The intensity ratio $\left(I_{\mathrm{D}} / I_{\mathrm{G}}\right)$ is characteristic of the extent of disorder present within in a material. For our samples, the $I_{\mathrm{D}} / I_{\mathrm{G}}$ ratios of the GO, GO-NIPs and GO-MIPs samples were calculated to be $0.94,1.09$ and 1.18 , respectively, reflecting increasing disorder. The $I_{\mathrm{D}} / I_{\mathrm{G}}$ value of the GO-MIPs was greater than that of the GO and GO-NIPs samples, which suggests a strong interaction between the MIPs and the GO sheet. The Dband of the GO-MIPs occurred at $1328 \mathrm{~cm}^{-1}$, which was downshifted by $17 \mathrm{~cm}^{-1}$ compared to that of GO. The Raman shifts of the D-band for the GO-MIPs provide evidence of charge transfer between the GO sheets and MIPs, indicating that the GO-MIPs composite had been successfully synthesized.

\subsection{Optimization of DSPE conditions}

In order to select the optimal DSPE conditions for the extraction of the cefadroxil, $100 \mathrm{mg}$ GO-MIPs particles and a series of sample solutions spiked with $1.0 \mu \mathrm{g} \mathrm{mL} \mathrm{mL}^{-1}$ standard mixture were used to study the extraction performance of the DSPE under different experimental conditions. In this experiment, several parameters, including the sample $\mathrm{pH}$, extraction time, and desorption conditions, were investigated to achieve the optimal extraction efficiency for FAD. All of the experiments were performed in triplicate, and the means of the results were used in the optimization evaluation.

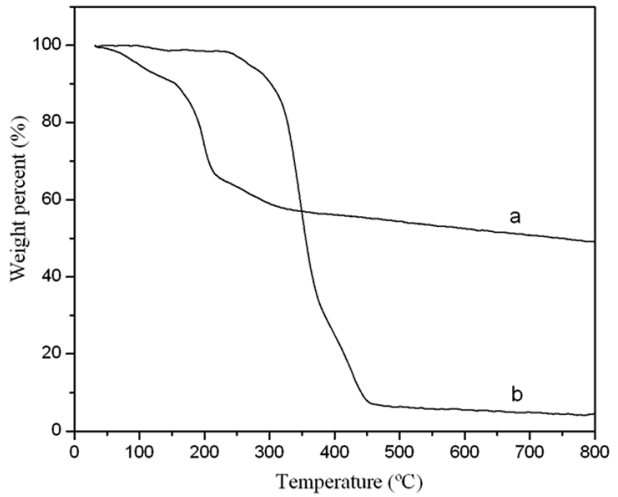

Fig. 6 The TGA curves of GO (a) and GO-MIPs composite (b). Conditions: heating rate, $10{ }^{\circ} \mathrm{C} \mathrm{min}^{-1}$ in nitrogen.

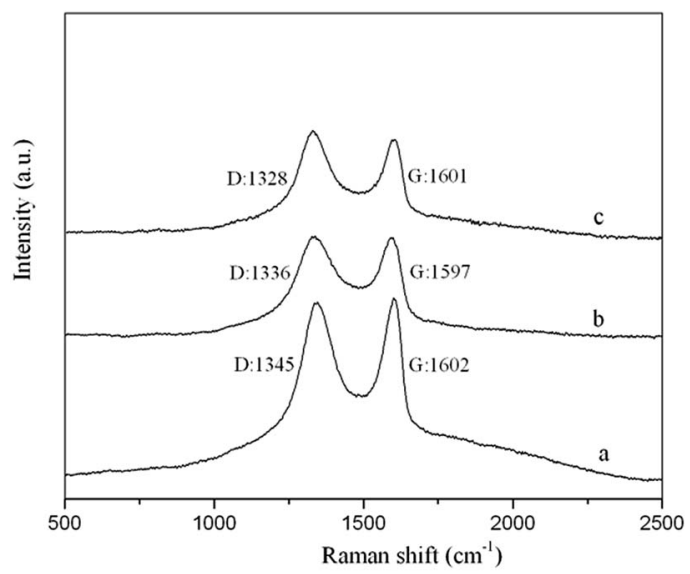

Fig. 7 The Raman spectra of the (a) GO (D band: 1345, G band: 1602); (b) GO-NIPs (D band: 1336, G band: 1597); (c) GO-MIPs (D band: 1328, G band: 1601)

3.2.1 Effect of the cephalosporin extract solution pH. The sample pH dramatically affects the extraction efficiency. A suitable $\mathrm{pH}$ of the sample solution can improve the extraction efficiency and reduce matrix interferences. The influence of the $\mathrm{pH}$ of the sample solution on the extraction efficiency was studied from $\mathrm{pH} 2.0$ to $\mathrm{pH} 12.0$ with $\mathrm{HCl}\left(1 \mathrm{~mol} \mathrm{~L}^{-1}\right)$ or $\mathrm{NaOH}$ $\left(1 \mathrm{~mol} \mathrm{~L}^{-1}\right)$. As shown in Fig. 8a, the extraction efficiency was highest when the $\mathrm{pH}$ was 6.0. However, when the $\mathrm{pH}$ was higher than 6.0, the extraction efficiency tended to decrease and no obvious change in the extraction efficiency of FAD was observed from $\mathrm{pH} 8.0$ to $\mathrm{pH}$ 12.0. The extraction efficiency dropped in strongly basic or acidic conditions, such pH-dependent behavior may be attributed to the hydrogen bonding interactions between the FAD and the GO-MIPs. Under acidic conditions, the FAD ( $\mathrm{p} K_{\mathrm{a}}=2.7, \mathrm{FAD}$ is neutral only within the narrow $\mathrm{pH}$ range of 5.0-6.0) will be protonated, this is not favorable for the formation of hydrogen bonds between the amino groups
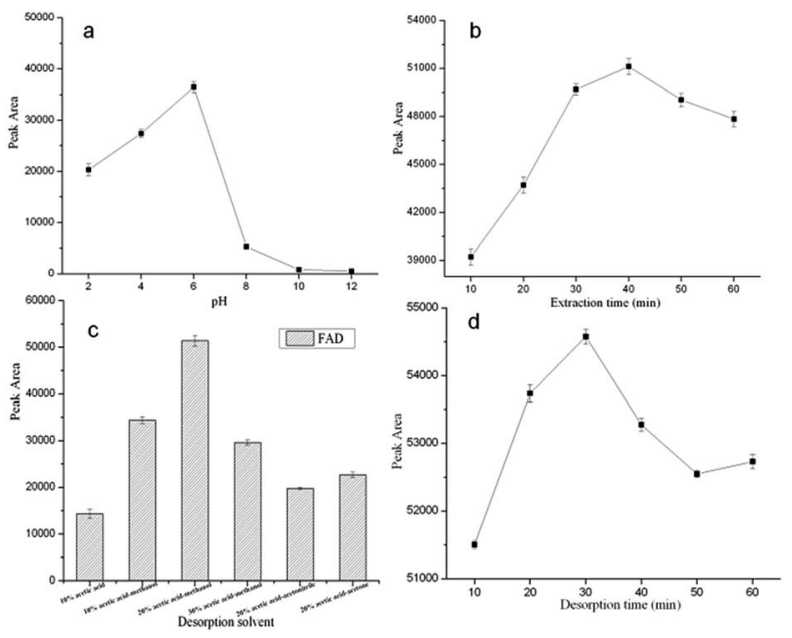

Fig. 8 Effects of sample pH (a) different extraction times (b) desorption reagents (c) and desorption times (d) on the peak areas of FAD extracted by the GO-MIPs-DSPE method. 


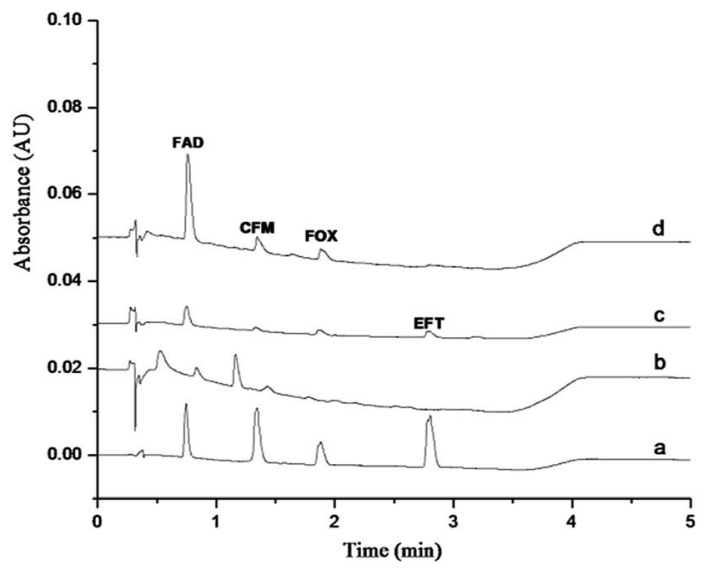

Fig. 9 Chromatogram of a $1 \mu \mathrm{g} \mathrm{mL} \mathrm{L}^{-1}$ standard solution injected directly (a), treated by GO-DSPE (b), GO-NIPs-DSPE (c), and GOMIPs-DSPE method (d).

and hydroxyl groups. Under alkaline conditions, the hydroxyl groups of the GO-MIPs would give priority to combination with the hydroxide in solution, resulting partially in the weakness of the hydrogen bonding interactions between the FAD and the GO-MIPs. Besides, $\pi-\pi$ interactions and hydrophobic interactions between the FAD and the GO-MIPs were favorable for keeping the extraction efficiency of the FAD in a strong acidic or basic media as well. Therefore, the sample matrix was adjusted to $\mathrm{pH} 6.0$ in the following experiments.

3.2.2 Effect of the extraction time. In the GO-MIPs-DSPE method, the maximum extraction of analytes is achieved at equilibrium. To obtain the highest extraction efficiency of FAD, the profile of the extraction time was investigated over 1060 min. As shown in Fig. 8b, the peak areas of the target analytes increased from $10 \mathrm{~min}$ to $40 \mathrm{~min}$ but decreased after $40 \mathrm{~min}$. This phenomenon might have resulted from FAD loss caused by the prolonged extraction time, which would be disadvantageous for the contact between FAD and the GO-MIPs particles. Therefore, $40 \mathrm{~min}$ was selected as the extraction time.

3.2.3 Effect of the desorption reagents. A series of desorption reagents including acetic acid-water $(10 / 90, \mathrm{v} / \mathrm{v})$, acetic acid-methanol $(10 / 90,20 / 80$ and 30/70, v/v), acetic acid-acetonitrile $(20 / 80, v / v)$, and acetic acid-acetone $(20 / 80, v / v)$ was investigated in GO-MIPs-DSPE-UPLC-PDA procedure. The FAD is easily dissolves in methanol. Acetic acid added to methanol would be beneficial to penetrate the GO-MIPs. It is beneficial for the eluent to come into sufficient contact with the adsorbed FAD. Furthermore, the acetic acid eluents are conducive to destroying the hydrogen bond between the FAD and GO-MIPs. The results indicated that acetic acid-methanol $(20 / 80, \mathrm{v} / \mathrm{v})$ as desorption solvent could obtain satisfactory extraction efficiency of FAD (Fig. 8c) and produce a clear chromatogram. Thus, acetic acid-methanol $(20 / 80, \mathrm{v} / \mathrm{v})$ was selected for subsequent experiments.

3.2.4 Effect of the desorption time. After extraction, the mixture was desorbed by acetic acid-methanol $(20 / 80, \mathrm{v} / \mathrm{v})$ via vortex mixing. A series of desorption times was investigated over 10-60 $\mathrm{min}$ at room temperature to ensure that mainly of the

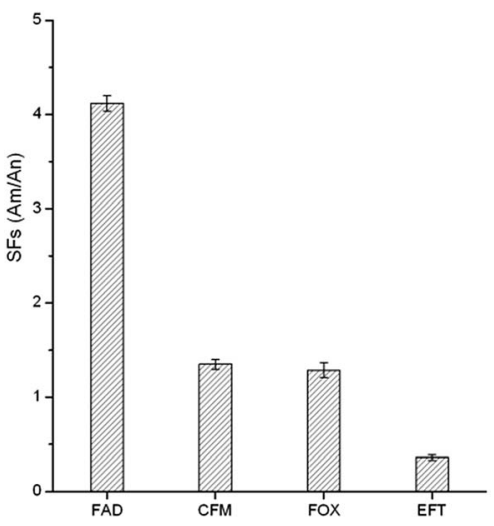

Fig. 10 Selectivity factors (SFs) of GO-MIPs.

FAD completely desorbed from the GO-MIPs particles. The highest peak area of FAD was achieved at 30 min (Fig. 8d), and the peak area of FAD decreased when desorption was performed for longer than $30 \mathrm{~min}$ because the desorbed analytes could have been reabsorbed by the GO-MIPs particles. Desorption was incomplete when a shorter desorption time was used. Therefore, $30 \mathrm{~min}$ was selected as the desorption time for the DSPE method.

\subsection{Comparison of GO-NIPs and GO-MIPs}

In this work, the GO-NIPs and GO-MIPs particles were used separately to extract a $1.0 \mu \mathrm{g} \mathrm{mL}^{-1}$ standard solution mixture via the proposed DSPE laboratory procedure, and the results are compared in Fig. 9. As shown in the figure, the four target analytes were slightly but clearly detected without any preparation (curve a) but were almost not detected when using the proposed DSPE procedure with the GO (curve b) and GO-NIPs particles (curve c). Compared with the GO-NIPs particles, the FAD peak area increased and the GO-MIPs particles exhibited advantages in the extraction process after the GO-MIPs-DSPE procedure (curve d). The comparison indicates that the GOMIPs particles exhibited better selective extraction than the GO-

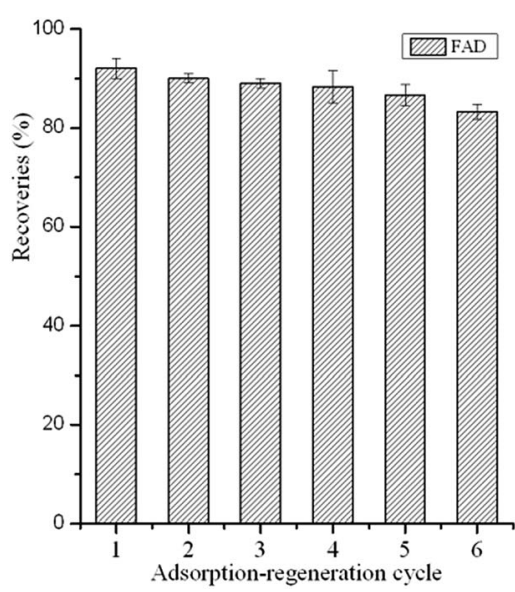

Fig. 11 Reusability of the GO-MIPs. 
NIPs particles. The matching pore size and recognition sites of the GO-MIPs improved the extraction selectivity for the target FAD. However, its enrichment effect is less obvious, which may be due to GO-MIPs particles on the surface of the formation of three-dimensional cavity is not too much. Non-specific adsorption was dominant for the GO-NIPs. Because the molecular structures of the other three cephalosporins were substantially different from the structure of FAD, these three cephalosporins were less recognized by the GO-MIPs particles with FAD as the template, decreasing the extraction selectivity for these three cephalosporins.

\subsection{Selectivity of the GO-MIPs}

To evaluate the selectivity of the GO-MIPs particles for the target FAD molecule, CFM, FOX, and EFT were chosen for comparison due to their modalities, structural similarities and coexistence with FAD in real samples. The selectivity was calculated using the selectivity factors (SFs): $\mathrm{SFs}=A_{\mathrm{M}} / A_{\mathrm{N}}$, where $A_{\mathrm{M}}$ and $A_{\mathrm{N}}$ are the peak areas obtained using the GO-MIPs and GO-NIPs particles treatments, respectively. As observed in Fig. 10, the GO-MIPs particles exhibited the highest SFs for FAD, with an average value of 4.12 and a relative standard deviation (RSD) of $8.2 \%(n=3)$, whereas the average SFs for CFM, FOX and EFT were $1.35(\mathrm{RSD}=4.8 \%, n=3), 1.29(\mathrm{RSD}=$ $8.3 \%, n=3$ ), and 0.36 ( $\mathrm{RSD}=3.2 \%, n=3$ ), respectively. The different selectivity may be due to the combination of hydrogen bond, electrostatic interaction, $\pi-\pi$ stacking interaction, and distinct stereo-selectivity. The extraction selectivity for FAD of the GO-MIPs particles was clearly better than that for other three cephalosporins, indicating that the GO-MIPs particles showed stable adsorption and a greater binding capacity for FAD. This increased capacity may have been attributable to the perfect fit of the shapes of the cavities in the polymers with the unique molecular structure of FAD. Thus, CFM, FOX and EFT cannot be adsorbed into the imprinted cavities via specific binding. These values, which are superior to unity, are proof of the specific interactions between FAD and the GO-MIPs particles. Therefore, the GO-MIPs particles showed good selectivity for the template molecule.

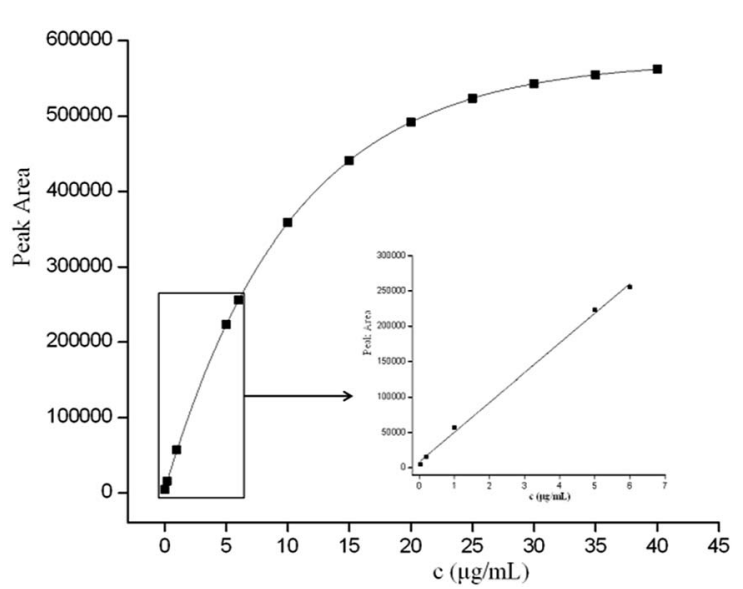

Fig. 12 Peak areas of different concentrations of FAD and the linearity curve (inset).

\subsection{Reusability of the GO-MIPs}

To investigate the reusability of the GO-MIPs particles, consecutive adsorption-regeneration cycles were performed with the same GO-MIPs particles. After each cycle, the GO-MIPs particles were consecutively regenerated with $15 \mathrm{~mL}$ of acetic acid-methanol $(20 / 80, \mathrm{v} / \mathrm{v})$ and $5 \mathrm{~mL}$ of methanol under vortex conditions. The substantial amounts of solvents were used to reduce carryover effects and interferences between each adsorption-regeneration process. The first desorption was used to investigate the carryover effects of the FAD. No FAD was detected. The GO-MIPs particles have been shown to be reusable up to 13 times. The first six adsorption-regeneration results are shown in Fig. 11. The results demonstrated that the GOMIPs particles are stable and may become an ideal candidate for pretreatment of aqueous samples.

\subsection{Evaluation of the GO-MIPs-DSPE method}

The performance of the optimized GO-MIPs-DSPE method was evaluated using UPLC-PDA. Quantitative parameters including the linear range, linear equations, correlation coefficients, limit of detection (LOD), and limit of quantification (LOQ) were evaluated for the extraction of FAD under the optimized extraction conditions. Calibration standard solutions of $0.04 \mu \mathrm{g}$ $\mathrm{mL}^{-1}$ to $6.0 \mu \mathrm{g} \mathrm{mL}^{-1}$ were extracted using the GO-MIPs-DSPE method and analyzed by UPLC. A good linear relationship with a good correlation coefficient $\left(R^{2}=0.9979\right)$ was obtained. Moreover, the linear regression equation for FAD was $y=$ $42061 x+8219$, where $y$ represents the peak area of FAD and $x$ denotes the FAD concentration in the standard solution. The LOD and LOQ of FAD were determined in standard solutions to be $0.01 \mu \mathrm{g} \mathrm{mL}{ }^{-1}$ and $0.03 \mu \mathrm{g} \mathrm{mL}^{-1}$, calculated as signal-to-noise ratios of $3(\mathrm{~S} / \mathrm{N}=3)$ and $10(\mathrm{~S} / \mathrm{N}=10)$, respectively. As shown in Fig. 12, as the concentration increased beyond the linear range, the peak areas slowly increased, indicating that the material had achieved saturation (maximum saturated adsorption amount is approximately $\left.35 \mu \mathrm{g} \mathrm{mL}{ }^{-1}\right){ }^{40}$

The precision of the GO-MIPs-DSPE-UPLC method was investigated by performing intra- and inter-day assays. The intra-assay precision was measured from six continuous injections performed on the same day, and the inter-day precision was calculated from measurements performed on three consecutive days. The RSD of the FAD peak area was $4.5 \%$ and $5.9 \%$ in the intra- and inter-assay precision assays, respectively.

Table 1 Recoveries from real environmental water samples (river water and tap water, $n=3$ )

\begin{tabular}{|c|c|c|c|c|}
\hline $\begin{array}{l}\text { Analyte } \\
\text { (FAD) }\end{array}$ & $\begin{array}{l}\text { Real sample } \\
\left(\mu \mathrm{g} \mathrm{mL} \mathrm{mL}^{-1}\right)\end{array}$ & $\begin{array}{l}\text { Concentration added } \\
\left(\mu \mathrm{g} \mathrm{mL}^{-1}\right)\end{array}$ & $\begin{array}{l}\text { Average } \\
\text { recovery (\%) }\end{array}$ & $\begin{array}{l}\text { RSD } \\
(\%)\end{array}$ \\
\hline \multirow[t]{4}{*}{ River water } & \multirow[t]{4}{*}{ Not detected } & 0.2 & 104.8 & 10.1 \\
\hline & & 1.0 & 80.6 & 4.9 \\
\hline & & 5.0 & 79.6 & 6.3 \\
\hline & & 0.2 & 102.4 & 8.1 \\
\hline \multirow[t]{2}{*}{ Tap water } & \multirow[t]{2}{*}{ Not detected } & 1.0 & 72.5 & 6.1 \\
\hline & & 5.0 & 78.5 & 7.9 \\
\hline
\end{tabular}


Table 2 Comparison of proposed method with other methods applied for $\mathrm{FAD}^{a}$

\begin{tabular}{|c|c|c|c|c|c|c|c|c|}
\hline Sample & $\begin{array}{l}\text { Sample } \\
\text { preparation }\end{array}$ & $\begin{array}{l}\text { Detection } \\
\text { method }\end{array}$ & $\begin{array}{l}\text { Linear range } \\
\left(\mu \mathrm{g} \mathrm{mL} L^{-1}, \mu \mathrm{g} \mathrm{g}^{-1}\right)\end{array}$ & $R^{2}$ & $\begin{array}{l}\text { LOD } \\
\left(\mu \mathrm{gL}^{-1}, \mu \mathrm{g} \mathrm{g}^{-1}\right)\end{array}$ & Recovery (\%) & RSD (\%) & Ref. \\
\hline Milk & SPME & HPLC-PDA & $0.1-10$ & 0.9995 & 0.02 & $83-92$ & $5.5-8.3$ & 37 \\
\hline Milk & MISPE & HPLC-UV & - & - & - & $>60$ & - & 28 \\
\hline Milk & MSPD & HPLC-PDA & $0.0192-0.24$ & 0.9984 & 0.0063 & $93.3-103.9$ & $1.2-6.1$ & 42 \\
\hline Milk & MSPD & HPLC-UV & $0.0462-0.2$ & 0.9916 & 0.0462 & $93.8-101.9$ & $2.0-5.7$ & 43 \\
\hline Water & - & SIA-PC & $1-10$ & 0.9969 & 0.0178 & $100.2-101.7$ & $0.57-1.98$ & 44 \\
\hline Water & SPE & CZE-LIF & $0.03-0.6$ & 0.9990 & 0.0075 & $88.6-96.6$ & $0.82-8.15$ & 45 \\
\hline Water & DSPE & UPLC-PDA & $0.04-6.0$ & 0.9979 & 0.01 & $72.5-104.8$ & $4.5-5.9$ & Thi \\
\hline
\end{tabular}

${ }^{a}$ SPME solid-phase microextraction, MSPD matrix solid-phase dispersion extraction, HPLC-PDA high-performance liquid chromatographyphotodiode array, HPLC-UV high-performance liquid chromatography-ultraviolet detection, SIA-PC sequential injection analysisspectrophotometric, CZE-LIF capillary zone electrophoresis-laser-induced fluorescence detection.

These data indicate that the proposed method is acceptable and stable for the selective extraction of FAD.

\subsection{Real samples}

To assess the accuracy of the established analytical method, real river and tap water samples were analyzed using the standard addition method. The filtered samples were spiked with cephalosporins at three different concentrations of $0.2 \mu \mathrm{g} \mathrm{mL}^{-1}, 1.0$ $\mu \mathrm{g} \mathrm{mL}^{-1}$ and $5.0 \mu \mathrm{g} \mathrm{mL} \mathrm{m}^{-1}$ and were subsequently extracted using the optimized method.

For the river water samples, the recoveries ranged from $79.6 \%$ to $104.8 \%$, and the RSDs $(n=3)$ ranged from $4.9 \%$ to $10.1 \%$. For the tap water samples, the recoveries ranged from $72.5 \%$ to $102.4 \%$, and the RSDs $(n=3)$ ranged from $6.1 \%$ to $8.1 \%$ (Table 1). These satisfactory recoveries indicate negligible effects of coexisting species and matrix components in the environmental water samples.

\subsection{Comparison of the proposed method with previous reports}

A comparison of the developed method with other methods for the extraction of FAD in water and other samples is presented in Table 2, which shows significant advantages of the proposed method in the extraction and determination of FAD from environmental water samples. A satisfactory linearity range, correlation coefficient, LOD, recovery and precision were obtained with the proposed GO-MIPs-DSPE-UPLC method compared to other methods. ${ }^{\mathbf{4 1 4 5}}$ The GO-MIPs particles exhibited an excellent selective adsorption capacity. Thus, GOMIPs particles-based DSPE could be used as a novel, simple and easy method for the effective extraction of analytes from complex matrices.

\section{Conclusions}

In the current study, GO-MIPs particles were successfully synthesized for FAD extraction using a novel imprinting technique, and a DSPE method based on GO-MIPs particles combined with UPLC-PDA was developed for the determination of FAD in water samples. GO nanosheets are suitable supports for MIPs because of their high surface area and 2D layered structure with a variety of oxygen-containing groups. The GOMIPs particles exhibited a high adsorption capacity, high selectivity, and rapid binding activity for FAD because of the combination of the large surface area of GO and the selectivity of the MIPs. Under the optimized conditions, this method demonstrated a low LOD and satisfactory repeatability but also exhibited certain disadvantages, such as a narrow linear range, because the material reached maximum adsorption. The synthesized GO-MIPs particles could be used for the separation and removal of FAD from environmental water samples. In conclusion, the newly developed method provides not only a simple, fast, accurate, reliable and sensitive analytical procedure but also a great potential adsorbent for the removal of FAD from more complex samples.

\section{Acknowledgements}

This work was financially supported by the Beijing Natural Science Foundation (No. 2162008), the Beijing Municipal Science and Technology Commission (No. Z141100002614020) and the Importation and Development of High-Caliber Talents Project of Beijing Municipal Institutions (No. CIT\&TCD 201504083).

\section{References}

1 D. Liu, N. Z. Song, W. Feng and Q. Jia, RSC Adv., 2016, 6, 11742-11748.

2 E. N. Evaggelopoulou and V. F. Samanidou, Food Chem., 2013, 136, 1322-1329.

3 F. J. Lara, M. del Olmo-Iruela, C. Cruces-Blanco and C. Quesada-Molina, TrAC, Trends Anal. Chem., 2012, 38, 5266.

4 A. M. Yehia, R. M. Arafa, S. S. Abbas and S. M. Amer, Spectrochim. Acta, Part A, 2016, 153, 231-240.

5 P. M. Shah, Int. J. Antimicrob. Agents, 2002, 19, 163-164. 
6 J. D. Williams, K. G. Naber, A. Bryskier, N. Høiby, I. M. Gould, P. Periti, H. Giamarellou and B. Rouveix, Int. J. Antimicrob. Agents, 2001, 17, 443-450.

7 S. A. E. Kools, J. F. Moltmann and T. Knacker, Regul. Toxicol. Pharmacol., 2008, 50, 59-65.

8 S. Cox, A. Martin, M. Hamill, N. Pistole, J. Steeil and J. Schumacher, Biomed. Chromatogr., 2014, 28, 1126-1130.

9 R. Pérez-Burgos, E. M. Grzelak, G. Gokce, J. Saurina, J. Barbosa and D. Barron, J. Chromatogr. B: Biomed. Sci. Appl., 2012, 899, 57-65.

10 W. H. Lu, W. N. Ming, X. S. Zhang and L. X. Chen, Electrophoresis, 2016, 37, 2487-2495.

11 X. Q. Wu, X. Y. Wang, W. H. Lu, X. R. Wang, J. H. Li, H. Y. You, H. Xiong and L. X. Chen, J. Chromatogr. A, 2016, 1435, 30-38.

12 W. Liu, Z. J. Zhang and Z. Q. Liu, Anal. Chim. Acta, 2007, 592, 187-192.

13 K. S. Kim, Y. Zhao, H. Jang, S. Y. Lee, J. M. Kim, K. S. Kim, J. H. Ahn, P. Kim, J. Y. Choi and B. H. Hong, Nature, 2009, 457, 706-710.

14 J. L. Shen, X. Xin, Y. J. Zhang, L. F. Song, L. Wang, W. Y. Tang and Y. J. Ren, Carbohydr. Polym., 2015, 117, 592-599.

15 S. Stankovich, D. A. Dikin, G. H. Dommett, K. M. Kohlhaas, E. J. Zimney, E. A. Stach, R. D. Piner, S. T. Nguyen and R. S. Ruoff, Nature, 2006, 442, 282-286.

16 Y. Y. Wen, Z. L. Niu, Y. L. Ma, J. P. Ma and L. X. Chen, J. Chromatogr. A, 2014, 1368, 18-25.

17 R. Z. Wang, D. L. Huang, Y. G. Liu, Z. W. Peng, G. M. Zeng, C. Lai, P. Xu, C. Huang, C. Zhang and X. M. Gong, RSC Adv., 2016, 6, 106201-106210.

18 N. S. Ye and J. Li, J. Sep. Sci., 2014, 37, 2239-2247.

19 H. M. Qiu, C. N. Luo, M. Sun, F. G. Lu, L. L. Fan and X. J. Li, Carbon, 2012, 50, 4052-4060.

20 M. Y. Yen, M. C. Hisao, S. H. Liao, P. I. Liu, H. M. Tsai and C. C. M. Ma, Carbon, 2011, 49, 3597-3606.

21 C. Y. Shi, J. R. Meng and C. H. Deng, J. Mater. Chem., 2012, 22, 20778-20785.

22 O. I. Parisi, C. Morelli, F. Puoci, C. Saturnino, A. Caruso, D. Sisci, G. E. Trombino, N. Piccia and M. S. Sinicropi, J. Mater. Chem. B, 2014, 2, 6619-6625.

23 B. Pluhar, U. Ziener and B. Mizaikoff, J. Mater. Chem. B, 2015, 3, 6248-6254.

24 Y. H. Wang, H. M. Duan, L. L. Li, X. J. Wang, J. B. Li, Y. A. Bu and C. A. Luo, RSC Adv., 2016, 6, 12391-12397.
25 M. J. Lerma-García, M. Zougagh and A. Ríos, Microchim. Acta, 2013, 180, 363-370.

26 Z. Terzopoulou, M. Papageorgiou, G. Z. Kyzas, D. N. Bikiaris and D. A. Lambropoulou, Anal. Chim. Acta, 2016, 913, 63-75.

27 Y. L. Hu, Y. F. Fan and G. K. Li, J. Chromatogr. A, 2012, 1228, 205-212.

28 C. Quesada-Molina, B. Claude, M. del Olmo-Iruela and P. Morin, Food Chem., 2012, 135, 775-779.

29 F. J. Ning, T. T. Qiu, Q. Wang, H. L. Peng, Y. B. Li, X. Q. Wu, Z. Zhang, L. X. Chen and H. Xiong, Food Chem., 2017, 221, 1797-1804.

30 S. Sharif, I. U. Khan, M. Ashfaq, M. S. Iqbal and S. Ahmad, J. Anal. Chem., 2010, 65, 1029-1034.

31 M. Javanbakht, M. M. Moein and B. Akbari-adergani, J. Chromatogr. B: Biomed. Sci. Appl., 2012, 911, 49-54.

32 M. Zarejousheghani, M. Möder and H. Borsdorf, Anal. Chim. Acta, 2013, 798, 48-55.

33 L. X. Chen, X. Y. Wang, W. H. Lu, X. Q. Wu and J. H. Li, Chem. Soc. Rev., 2016, 45, 2137-2211.

34 J. X. Liu, Q. L. Deng, D. Y. Tao, K. G. Yang, L. H. Zhang, Z. Liang and Y. K. Zhang, Sci. Rep., 2014, 4, 5487-5492.

35 Y. M. Liang, L. L. Yu, R. Yang, X. Li, L. B. Qu and J. J. Li, Sens. Actuators, B, 2017, 240, 1330-1335.

36 J. H. Xu, Y. Z. Wang and S. S. Hu, Microchim. Acta, 2017, 184, $1-44$.

37 J. X. Guo, Y. Z. Wang, Y. J. Liu, C. J. Zhang and Y. G. Zhou, Talanta, 2015, 144, 411-419.

38 K. Hemmati, R. Sahraei and M. Ghaemy, Polymer, 2016, 101, 257-268.

39 S. D. Pan, X. H. Chen, X. P. Li, M. Q. Cai, H. Y. Shen, Y. G. Zhao and M. C. Jin, J. Chromatogr. A, 2015, 1422, 1-12.

40 N. S. Ye, T. Gao and J. Li, Anal. Methods, 2014, 6, 7518-7524.

41 N. Rahim, S. B. S. Naqvi, M. Alam, E. Iqbal and R. Bibi, Trop. J. Pharm. Res., 2014, 13, 975-979.

42 E. G. Karageorgou, V. F. Samanidou and I. N. Papadoyannis, J. Sep. Sci., 2012, 35, 2599-2607.

43 E. G. Karageorgou and V. F. Samanidou, J. Sep. Sci., 2010, 33, 2862-2871.

44 J. Makchit, S. Upalee and C. Thongpoon, Anal. Sci., 2006, 22, 591-597.

45 A. H. Rageh, K. F. Klein and U. Pyell, Chromatographia, 2016, 79, 225-241. 